Short Communication

\title{
Allergin-1 on mast cells suppresses house dust mite-induced airway hyperresponsiveness in mice
}

Kaori Hitomi ${ }^{1}$, Satoko Tahara-Hanaoka ${ }^{1,2, *}$, Haruka Miki ${ }^{1,3}$, Kanako Iwata ${ }^{1}$, Shiro Shibayama ${ }^{4}$, Masato Kubo ${ }^{5,6}$, and Akira Shibuya ${ }^{1,2}$

${ }^{1}$ Department of Immunology

${ }^{2}$ Life Science Center of Tsukuba Advanced Research Alliance (TARA) and

${ }^{3}$ Department of Internal Medicine, Faculty of Medicine, University of Tsukuba, 1-1-1

Tennodai, Tsukuba, Ibaraki 305-8575, Japan.

${ }^{4}$ Research Center of Immunology, Tsukuba Institute, ONO Pharmaceutical Co., LTD. 17-2 Wadai, Tsukuba, Ibaraki 300-4247, Japan

${ }^{5}$ Division of Molecular Pathology, Research Institute for Biomedical Science, Tokyo University of Science, 2669 Yamazaki, Noda, Chiba, 278-0022, Japan

${ }^{6}$ Laboratory for Cytokine Regulation, RIKEN Center for Integrative Medical Sciences (IMS), 1-7-22 Suehiro-cho, Tsurumi-ku, Yokohama, Kanagawa 230-0045, Japan

*Corresponding should be addressed to Satoko Tahara-Hanaoka, PhD.,

Tel./fax: +81-29 $8533281 / 3410$.,

E-mail: tokothr@md.tsukuba.ac.jp

Running title: Allergin-1 inhibits HDM-induced AHR

Key words: asthma 


\begin{abstract}
Although airway hyperresponsiveness (AHR) is a prominent feature of asthma, how it is regulated remains incompletely understood. Allergin-1, an inhibitory immunoglobulin-like receptor containing an immunoreceptor tyrosine-based inhibitory motif (ITIM), is expressed on human and mouse mast cells (MCs) and inhibits high affinity receptor for IgE (FceRI)-mediated signaling. Using MC-deficient $\mathrm{Kit}^{\mathrm{W}-\mathrm{sh} / \mathrm{W}-\mathrm{sh}}$ mice and Mas-TRECK mice, which carries a diphtheria toxin (DT)-induced MC-deletion system based on il4 enhancer elements, we demonstrate here that MCs are involved in the induction of house dust mite (HDM)-induced AHR. Further, we show that MCs deficient in Allergin-1 exacerbated HDM-induced AHR, but had no effect on airway inflammation. In vitro analysis demonstrated that Allergin-1 inhibited anti-HDM allergen antibody-dependent HDM allergen-mediated degranulation by MCs. Thus, Allergin-1 on MCs plays an important role in the regulation of HDM-induced AHR.
\end{abstract}




\section{Introduction:}

Airway hyperresponsiveness (AHR) causing airway obstruction is a prominent feature of asthma. Mast cells (MCs) infiltrated into the airways play an important role in AHR (1). MCs express the high-affinity receptor for $\operatorname{IgE}(\mathrm{Fcc} R \mathrm{R})$ that binds to an allergen through FceRI-bound IgE. This interaction activates MCs, resulting in the secretion of a broad spectrum of pro-inflammatory cytokines, chemokines, and chemical mediators (2). IgE production by B cells is promoted by T helper 2 (Th2)-derived cytokines, IL-4, and anti-IgE therapy is beneficial in certain patients with severe asthma (3), suggesting that IgE-mediated signals in MCs play an important role in the pathogenesis of asthma. However, the role of MCs in the development of AHR is somewhat controversial (4), and the precise mechanisms by which MCs regulate AHR remain to be elucidated.

Allergin-1, which is encoded by Milr1, is an inhibitory immunoglobulin-like receptor expressed on both human and mouse MCs $(5,6)$. When Allergin-1 is co-ligated with IgE-bound FceRI, tyrosine residues of immunoreceptor tyrosine-based inhibitory motif (ITIM) in the cytoplasmic region of Allergin-1 are phosphorylated and recruits the Src homology 2 domain-containing tyrosine phosphatases (SHP-1) and SHP-2, thereby blocking an early step in the activation signal mediated by FceRI (5). Allergin-1-deficient mice show significantly enhanced passive systemic and cutaneous anaphylaxis, indicating that Allergin-1 suppresses IgE-mediated $\mathrm{MC}$ activation in vivo (5). Allergin-1 also inhibits toll-like receptor (TLR) 2-mediated activation of MCs in the skin and suppresses dermatitis (7).

In this study, we examined the role of Allergin-1 on MCs in AHR and Th2 responses in mice. We show that Allergin-1 regulated MC activation and suppressed AHR, but not Th2 responses, induced by house dust mite (HDM). 


\section{Materials and Methods}

\section{Animals}

Female BALB/cAJcl and C57BL/6N wild-type (WT) mice (8 to 12 weeks old) were purchased from Clea Japan (Tokyo, Japan). Allergin-1-deficient $\left(M i l r 1^{-/}\right)$mice on the BALB/c background were generated by backcrossing Milr1 ${ }^{-/}$C57BL/6N mice (5) to the BALB/cAJcl genetic background for 12 generations. $K i t^{W-s h / W-s h}$ mice were provided by Riken BioResource Center (Tsukuba, Japan). Mas-TRECK mice on the BALB/c background have been previously described (8). All procedures were done according to the guidelines of the animal ethics committee of the University of Tsukuba (permit number 17-284).

\section{MC engraftment}

To generate bone marrow (BM)-derived cultured MCs (BMMCs), $2 \times 10^{6} \mathrm{BM}$ cells were cultured in a 10-cm dish in complete RPMI 1640 medium (Sigma-Aldrich, St. Louis, MO, USA) containing 10\% FBS (Life Technologies, Auckland, New Zealand) in the presence of $10 \mathrm{ng} / \mathrm{mL}$ stem cell factor (R\&D Systems, Minneapolis, MN, USA) and $4 \mathrm{ng} / \mathrm{mL}$ IL-3 (R\&D Systems) for 4-5 weeks, at which time the cell population was composed of $>95 \% \mathrm{MCs}$, as assessed with flow cytometry. Mas-TRECK mice were injected with diphtheria toxin (DT, $250 \mathrm{ng}$; Sigma-Aldrich) intraperitoneally 5 consecutive days every 2 weeks to deplete endogenous MCs. On day 7 after the first injection of DT, $1 \times 10^{7} \mathrm{WT}$ or Milr $^{-/}$mice-derived BMMCs were injected intravenously into each DT-treated Mas-TRECK mouse. MCs reconstitution was assessed by flow cytometric analysis of peritoneal exudate MCs at 3 months after BMMCs injection. 
HDM-induced allergic asthma model

HDM allergen whole body extract (LSL Co., Ltd. Tokyo, Japan), derived from Dermatophagoides pteronyssinus (Der p), was used to induce allergic asthma. Animals were treated intranasally with $10 \mu \mathrm{g}$ of HDM extract 5 consecutive days a week for 4 weeks. Mice were assessed for airway hyperresponsiveness (AHR), airway inflammation, and serum $\operatorname{IgE}$ concentration $48 \mathrm{~h}$ after the last intranasal challenge.

Measurement of airway hyperresponsiveness (AHR) to methacholine

Airway resistances to different doses of aerosolized methacholine was measured by the computer-controlled piston ventilator system (FlexiVent, Montreal, Quebec, Canada) in accordance with the manufacturer's instructions. Briefly, $48 \mathrm{hr}$ after the last HDM challenge, mice were anesthetized, tracheotomized, and intubated intratracheally and exposed to an aerosol of $70 \mu \mathrm{l}$ PBS (basal readings) and then of $70 \mu \mathrm{l}$ methacholine in PBS at indicated concentration. Respiratory resistances were determined as the percentage change from the baseline index.

\section{Antibodies}

A monoclonal antibody (mAb) specific to mouse Allergin-1 (TX83; mouse IgG1) was generated in our laboratory, as described previously (5). TX83 was biotinylated with Biotinylation Kit (Sulfo-OSu; DOJIN, Tokyo, Japan). MAbs to mouse CD45.2 (104), c-Kit (2B8), Ly6G (1A8), CD11b (M1/70), CD11c (HL3), and Siglec-F (E50-2440) were purchased from BD Biosciences (San Joes, CA, USA); mAbs against CD16/32 (2.4G2), FceRI (MAR-1), CD3 $\varepsilon$ (145-2C11), and B220 (RA3-6B2) were purchased 
from TONBO Bioscience (SanDiego, CA, USA); mAb against Gr-1 (RB6-8C5) and CD107a (1D4B) was purchased from BioLegend (SanDiego, CA, USA).

Flow cytometric analysis of the lung and bronchoalveolar lavage fluid (BALF) cells

The lung tissues were perfused with $1 \mathrm{ml}$ of phosphate-buffered saline (PBS) through the right ventricle, removed, and digested with collagenase II (300 U/sample, Worthington Biochemical, Lakewood, NJ, USA) at $37^{\circ} \mathrm{C}$ for $1 \mathrm{~h}$ and dissociated with a gentleMACS Dissociator (B.01 program; Miltenyi Biotec, Auburn, CA). To assess the airway eosinophilia, bronchoalveolar lavage fluid (BALF) was harvested $48 \mathrm{~h}$ after the last HDM challenge and the trachea was cannulated with a $21 \mathrm{G}$ needle and plastic tube and washed with $1 \mathrm{~mL}$ of PBS 3 times. The cells were treated with anti-CD16/32 mAb to avoid binding to $\mathrm{Fc} \gamma \mathrm{R}$ on ice for $10 \mathrm{~min}$ prior to incubation with indicated combination of antibodies. Propidium iodide (PI, $1 \mu \mathrm{g} / \mathrm{ml}$; Sigma-Aldrich, St. Louis, MO, USA) was used to gate out dead cells. Cells were analyzed on a BD LSR Fortessa cell analyzer (BD Biosciences) and the FlowJo software program (Tree Star, San Carlos, CA, USA).

\section{Measurement of serum IgE concentration by ELISA}

For quantitation of serum levels of IgE, 96-well ELISA plates (NUNC Thermoscientific, Yokohama, Japan) were coated with rat anti-mouse IgE (catalog no. R35-72, BD Biosciences) diluted in $50 \mathrm{mM}$ sodium bicarbonate buffer ( $\mathrm{pH}$ 9.6) as a capture antibody. IgE was detected by using biotinylated anti-mouse IgE (catalog no. R35-118, BD Biosciences), followed by streptavidin-conjugated horseradish peroxidase (GE Healthcare Biosciences, Little Chalfont, UK). Mouse IgE (catalog no. C38-2, BD 
Biosciences) was used as a standard. All of these assays were performed in accordance with the manufacturers' instructions.

\section{Histology}

Forty-eight hours after the last HDM challenge, mice were anesthetized, and their lungs were harvested and fixed in 10\% neutral buffered formalin. Fixed lungs were embedded in paraffin and stained with hematoxylin-eosin (HE) and periodic acid-Schiff (PAF). The goblet cell hyperplasia was assessed by measuring \% area of the airway basement membrane that was positive for PAS staining in the total area of the airway basement membrane in randomly selected three bronchioles of each mice. Three mice per group were analyzed for the goblet cell hyperplasia. All images were acquired using a BZ-X710 microscope (Keyence, Osaka, Japan), and the data were analyzed using a BZ-H3 analyzer and Hybrid Cell Count (Keyence).

\section{Degranulation assay}

Degranulation was evaluated by a flow cytometric analysis of the cell-surface exposure of CD107a (LAMP-1). LAMP-1 is an intracellular protein found on granule membranes that becomes exposed on cell-surface upon degranulation (9). BMMCs were incubated for $20 \mathrm{~h}$ at $37^{\circ} \mathrm{C}$ with serum obtained from a naïve mouse or from HDM-immunized mice (in which the serum IgE level was as high as $1 \mu \mathrm{g} / \mathrm{ml}$ ) and then were stimulated for $30 \mathrm{~min}$ at $37^{\circ} \mathrm{C}$ with the indicated concentrations of HDM extract. BMMCs were then stained with allophycocyanin (APC)-conjugated anti-CD107 and analyzed for the proportion of $\mathrm{CD} 107 \mathrm{a}^{+}$cells among c-Kit ${ }^{+} \mathrm{Fc}_{\mathrm{RII}}{ }^{+}$cells by using flow cytometry. 
Statistics

Statistical analyses were performed with the GraphPad Prism 7 software (La Jolla, CA, USA) by using the unpaired Student's t-test or one-way or two-way ANOVA followed by Bonferroni's multiple comparison test as a post-test. A $P$ value less than 0.05 was considered statistically significant. 


\section{Results and discussion}

We first examined the involvement of MCs in HDM-induced AHR by using MC-deficient $K i t^{W-s h / W-s h}$ mice. WT and $\mathrm{Kit}^{W-s h / W-s h}$ mice were immunized intranasally with $10 \mu \mathrm{g}$ of HDM extract 5 consecutive days a week for 4 weeks (Fig. 1A). The airway eosinophilia and serum IgE level were comparable between the two groups (Fig. 1B). However, AHR to methacholine was significantly decreased in $K i t^{W-s h / W-s h}$ mice compared with that in WT mice (Fig. 1B), suggesting that MCs are responsible for HDM-induced AHR, but are dispensable for Th2 responses. To further demonstrate the function of MCs in HDM-induced AHR, we used diphtheria toxin (DT)-treated Mas-TRECK mice, an MC deletion system that uses Il4 enhancer elements (8). Although basophils are also depleted in DT-treated Mas-TRECK mice transiently, they quickly recovered within 3 days after the last DT treatment (8). In agreement with a previous report (8), flow cytometry did not detect MCs in the peritoneal cavity 2 days after the last injection of DT in Mas-TRECK mice (Fig. 1C and D). The DT-treated Mas-TRECK mice were reconstituted with WT bone marrow-derived cultured mast cells (BMMCs), and then repeatedly immunized with $10 \mu \mathrm{g}$ of HDM extract by intranasal injection (Fig. 1C). The reconstitution of MCs was confirmed by analyzing the $\mathrm{MC}$ population in the peritoneal cavity 3 months after the BMMC transfer (Fig 1D). Although DT-treated Mas-TRECK mice, which lack MCs, were resistant to methacholine-induced AHR, the mice that had received WT BMMCs developed AHR in response to the challenge with methacholine (Fig. 1E). Collectively, these results indicate that MCs are responsible for HDM-induced AHR.

We then examined the expression of Allergin-1 on MCs accumulated in the 
lungs after HDM treatment. We found that the lung MCs (identified as CD45.2 ${ }^{+}$in $^{-} \mathrm{CD} 11 \mathrm{~b}^{+}$Siglec-F ${ }^{-} \mathrm{Fc}_{\mathrm{RI}}{ }^{+} \mathrm{c}-\mathrm{Kit}^{+}$cells) highly expressed Allergin-1. In contrast, the lung eosinophils (indicated as CD45.2 ${ }^{+}$Siglec- $\mathrm{F}^{+} \mathrm{CD} 11 \mathrm{~b}^{\text {high }}$ cells) expressed undetectable level of Allergin-1 (Fig. 2A). The numbers of MCs in the lung were comparable between WT and $M i l r 1^{-/}$mice after HDM treatment (Fig. 2A). To investigate the role of Allergin-1 on MCs in HDM-induced AHR, we reconstituted DT-treated Mas-TRECK mice with BMMCs derived from either WT or Milr ${ }^{-1-}$ mice and treated them with HDM as shown in Fig. 1A. The reconstitution efficiency was comparable between WT and Milr1 $1^{-/}$BMMCs, as assessed by the MC population in the peritoneal cavity 3 months after the BMMC transfer (Fig. 2B). After the intranasal administration of HDM, the numbers of total cells $\left(\mathrm{CD} 45^{+}\right.$cells $)$and eosinophils $\left(\mathrm{CD} 45^{+} \mathrm{Gr}-1^{\text {low }}\right.$ Siglec-F $\mathrm{F}^{+} \mathrm{CD} 11 \mathrm{c}^{-}$cells) in the bronchoalveolar lavage fluid (BALF) and the serum IgE levels were induced equally in both mouse populations, and no striking differences in goblet cell hyperplasia were observed between DT-treated Mas-TRECK mice that had been reconstituted with WT or Milr1 ${ }^{-1-}$ BMMCs (Fig. 2C-E). However, those mice reconstituted with Milrl ${ }^{-1}$ BMMCs showed more severe AHR to methacholine than did those reconstituted with WT BMMCs (Fig. 2F). Collectively, these results indicate that Allergin-1 on MCs is not involved in HDM-induced Th2 responses but rather inhibits HDM-induced AHR.

AHR is considered to be triggered by chemical mediators, such as histamine, in the granules released from MCs (1). Therefore, we examined whether Allergin-1 inhibits HDM allergen-induced degranulation from MCs. Since HDM-specific mouse IgE or IgG antibodies were unavailable, we used sera from HDM-immunized mice, in which the serum IgE level was as high as $1 \mu \mathrm{g} / \mathrm{ml}$ for HDM-induced degranulation from 
MCs. WT or Milr ${ }^{-/-}$BMMCs were sensitized overnight with the sera from either naïve or HDM-immunized mice, stimulated with HDM extract for $30 \mathrm{~min}$, and then analyzed for CD107a expression on MCs (a marker of degranulation) by flow cytometry. After sensitization, Milr1 ${ }^{-/-}$BMMCs had a larger population of $\mathrm{CD} 107 \mathrm{a}^{+}$cells than did WT BMMCs upon $1,000 \mathrm{ng} / \mathrm{ml}$ of HDM stimulation (Fig. 3A and B). In contrast, HDM-induced CD107a expression was scarcely detected on either genotype of BMMCs sensitized with naïve serum, demonstrating HDM allergen-induced MC degranulation requires sensitization of MCs with HDM-immunized sera. These results suggest that Allergin-1 inhibited anti-HDM antibody-dependent degranulation of MCs, thus suppressing HDM allergen-induced AHR.

The contribution of MCs in AHR has been controversial (4), because most previous studies on this issue used MC-deficient $K i t^{W-s h / W-s h}$ or $K i t^{W / W-v}$ mice and a virtual allergen, such as ovalbumin (OVA) with or without aluminum salt adjuvant $(4,10)$. Those mice carry abnormalities due to $c$-Kit mutation in both hematopoietic and nonhematopoietic tissues as well as mast cells (11). Mas-TRECK mice used in this study show only the defect of MCs and basophils, and we reconstituted Mas-TRECK mice with BMMCs, thus more appropriate than mice with $c$-Kit mutation. Moreover, HDM is one of the commonest aeroallergens; more than $50 \%$ of allergic patients and up to $85 \%$ of asthmatics children are sensitized with HDM (12-14). Therefore, a mouse model using HDM as an allergen might be more appropriate for human asthma.

A recent study by using mice deficient in IgE or FceRIa demonstrated that HDM-induced AHR is independent of IgE and FceRIa (15). In the current study, we showed that MCs were required for HDM-induced AHR in vivo and that MCs activation was dependent on the serum of HDM-immunized, but not naïve, mice in vitro, 
suggesting that HDM-induced AHR was induced by MCs activated by anti-HDM antibody. However, we did not show the direct evidence for involvement of anti-HDM IgE antibody and FceRIa. Since MC degranulation is also induced by the IgG immune complexes (16), it is also possible that MCs activation was mediated by the Fc $\gamma \mathrm{RI}$, rather than FceRIa, on MCs in our model. Further studies are required for understanding the mechanisms of HDM-induced AHR.

We demonstrated that MCs play an important role in HDM-induced AHR. However, previous reports demonstrated that treatment with cysteine proteases, including papain, induce AHR, for which basophils and type 2 innate lymphoid cells (ILC2) are responsible $(17,18)$. Cysteine proteases promote IL-4 production from basophils and activates ILC2, which augment inflammation via production of inflammatory cytokines and chemokines (17). Thus, basophil and ILC2-dependent AHR are involved in innate immune responses, whereas MCs-dependent AHR requires antibodies against HDM as results of adaptive immune responses.

Because AHR is a fundamental pathology in asthma, control of AHR should be a therapeutic goal in the management of patients with asthma. Our findings show that $\mathrm{MC}$ is a key driver for AHR and that Allergin-1 on MCs plays a regulatory role in this process. 


\section{Funding}

This work was supported in part by grants provided by Japan Society for the Promotion of Science (KAKENHI) [grant numbers $15 \mathrm{H} 04862$ to S. T.-H. and $16 \mathrm{H} 06387$ to AS] and was funded by ONO Pharmaceutical Co., Ltd.

\section{Acknowledgements}

We thank S. Tochihara for secretarial assistance. K. H. performed most of the experiments and analyzed the data; H. M. and K. I. did experiments; S. S. and M. K. contributed to experimental design; S. T. - H. designed and did experiments, analyzed data and wrote the paper; and A. S. supervised the overall project and wrote the paper. 


\section{References}

1 Bradding, P., Walls, A. F., and Holgate, S. T. 2006. The role of the mast cell in the pathophysiology of asthma. The Journal of allergy and clinical immunology $117: 1277$.

Galli, S. J., Kalesnikoff, J., Grimbaldeston, M. A., Piliponsky, A. M., Williams, C. M., and Tsai, M. 2005. Mast cells as "tunable" effector and immunoregulatory cells: recent advances. Annu Rev Immunol 23:749.

3 Vichyanond, P. 2011. Omalizumab in allergic diseases, a recent review. Asian Pac J Allergy Immunol 29:209.

4 Lei, Y., Gregory, J. A., Nilsson, G. P., and Adner, M. 2013. Insights into mast cell functions in asthma using mouse models. Pulm Pharmacol Ther 26:532.

5 Hitomi, K., Tahara-Hanaoka, S., Someya, S., Fujiki, A., Tada, H., Sugiyama, T., Shibayama, S., Shibuya, K., and Shibuya, A. 2010. An immunoglobulin-like receptor, Allergin-1, inhibits immunoglobulin E-mediated immediate hypersensitivity reactions. Nat Immunol 11:601.

Nagai, K., Tahara-Hanaoka, S., Morishima, Y., Tokunaga, T., Imoto, Y., Noguchi, E., Kanemaru, K., Imai, M., Shibayama, S., Hizawa, N., Fujieda, S., Yamagata, K., and Shibuya, A. 2013. Expression and function of Allergin-1 on human primary mast cells. PLoS One 8:e76160.

7 Tsurusaki, S., Tahara-Hanaoka, S., Shibagaki, S., Miyake, S., Imai, M., Shibayama, S., Kubo, M., and Shibuya, A. 2016. Allergin-1 inhibits TLR2-mediated mast cell activation and suppresses dermatitis. Int Immunol 28:605.

8 Sawaguchi, M., Tanaka, S., Nakatani, Y., Harada, Y., Mukai, K., Matsunaga, Y., Ishiwata, K., Oboki, K., Kambayashi, T., Watanabe, N., Karasuyama, H., Nakae, S., Inoue, H., and Kubo, M. 2012. Role of Mast Cells and Basophils in IgE Responses and in Allergic Airway Hyperresponsiveness. J Immunol 188:1809.

9 Grutzkau, A., Smorodchenko, A., Lippert, U., Kirchhof, L., Artuc, M., and Henz, B. M. 2004. LAMP-1 and LAMP-2, but not LAMP-3, are reliable markers for activation-induced secretion of human mast cells. Cytometry A 61:62.

10 Williams, C. M. and Galli, S. J. 2000. Mast cells can amplify airway reactivity and features of chronic inflammation in an asthma model in mice. J Exp Med 192:455.

11 Rodewald, H. R. and Feyerabend, T. B. 2012. Widespread immunological functions of mast cells: fact or fiction? Immunity $37: 13$.

12 de Blay, F., Pauli, G., Velten, M., and Bessot, J. C. 1994. Influence of mite exposure on symptoms of mite-sensitive patients with asthma. The Journal of allergy and clinical immunology 93:136.

13 Bousquet, J. 2000. Global initiative for asthma (GINA) and its objectives. Clin Exp Allergy 30 Suppl 1:2.

14 Nelson, R. P., DiNicolo, R., FernandezCaldas, E., Seleznick, M. J., Lockey, R. F., and Good, R. A. 1996. Allergen-specific IgE levels and mite allergen exposure in children with acute asthma first seen in an emergency department and in nonasthmatic control subjects. Journal of Allergy and Clinical Immunology 98:258.

15 McKnight, C. G., Jude, J. A., Zhu, Z., Panettieri, R. A., Jr., and Finkelman, F. D. 2017. House Dust Mite-Induced Allergic Airway Disease Is Independent of IgE and FcepsilonRIalpha. Am J Respir Cell Mol Biol 57:674.

16 Yu, Y., Blokhuis, B. R., Garssen, J., and Redegeld, F. A. 2016. Non-IgE mediated mast cell activation. Eur J Pharmacol 778:33.

17 Motomura, Y., Morita, H., Moro, K., Nakae, S., Artis, D., Endo, T. A., Kuroki, Y., Ohara, O., Koyasu, S., and Kubo, M. 2014. Basophil-derived interleukin-4 controls the function of natural helper cells, a member of ILC2s, in lung inflammation. Immunity 40:758.

18 Gregory, L. G. and Lloyd, C. M. 2011. Orchestrating house dust mite-associated allergy in the lung. Trends Immunol 32:402. 
Figure 1

A

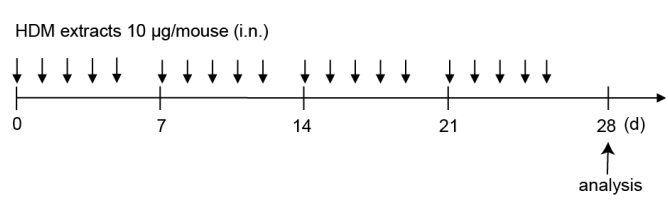

B
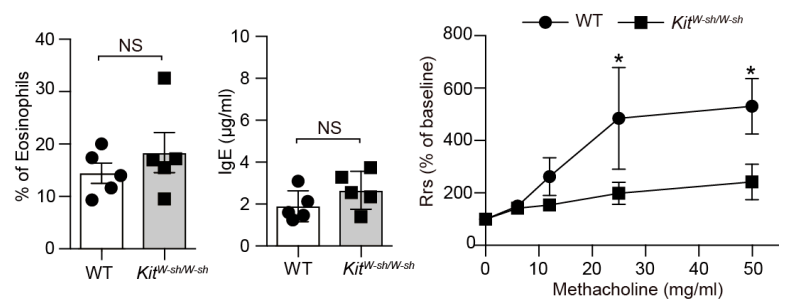

C

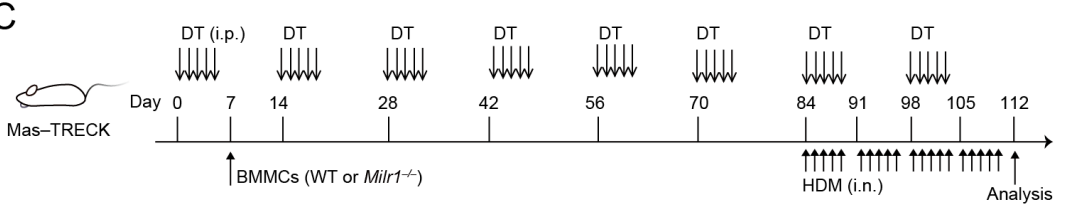

D
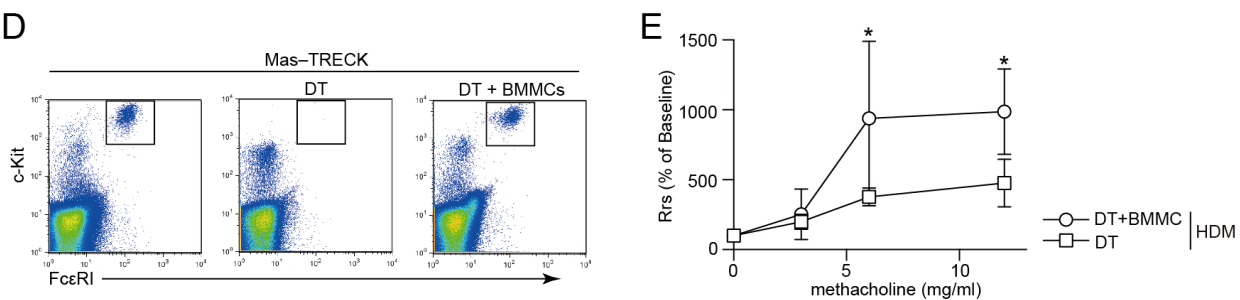

Figure 1. AHR in MC-deficient mice. (A and B) C57BL/6N WT $(n=4)$ and MC-deficient $K i t^{W-s h / W-s h}$ mice $(n=4)$ were immunized intranasally with $10 \mu \mathrm{g}$ of HDM extract 5 consecutive days a week for 4 weeks. (A) The experimental design (B) The proportion of eosinophils $\left(\mathrm{CD} 45^{+} \mathrm{CD} 11 \mathrm{c}^{-}\right.$Siglec- $\left.\mathrm{F}^{+}\right)$in the bronchoalveolar lavage fluid (BALF), serum IgE level, and AHR to methacholine were analyzed at $48 \mathrm{~h}$ after the last HDM immunization. (C-E) The experimental design (C). Three months after the BMMC injection, peritoneal exudate MCs in the naïve state from DT-non-treated, DT-treated (DT), and BMMC-reconstituted DT-treated (DT + BMMC) Mas-TRECK mice were analyzed by flow cytometry (D). DT-treated Mas-TRECK mice given no BMMCs (DT; $n=4)$ or reconstituted with WT-derived BMMCs (DT + BMMCs; $n=5)$ were immunized intranasally with $10 \mu \mathrm{g}$ of HDM extract. AHR to methacholine was analyzed at $48 \mathrm{~h}$ after the last HDM immunization (E). Rrs, respiratory resistance. An 
unpaired $t$-test was used to determine significant differences between the two groups. N.S., not significant. *, $P<0.05$. Data are representative of at least 2 independent experiments (mean \pm s.e.m.). 
Figure 2
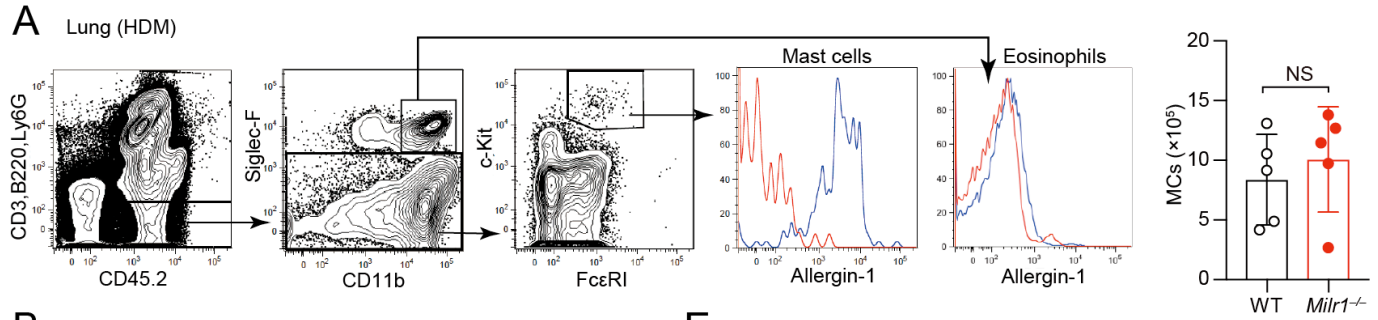

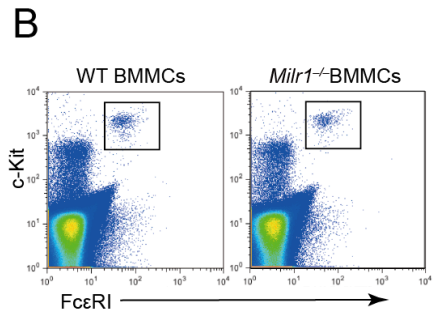

C Pl-

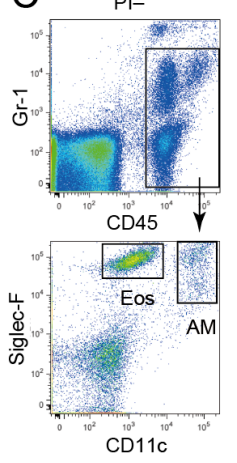

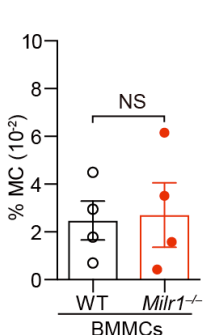

E

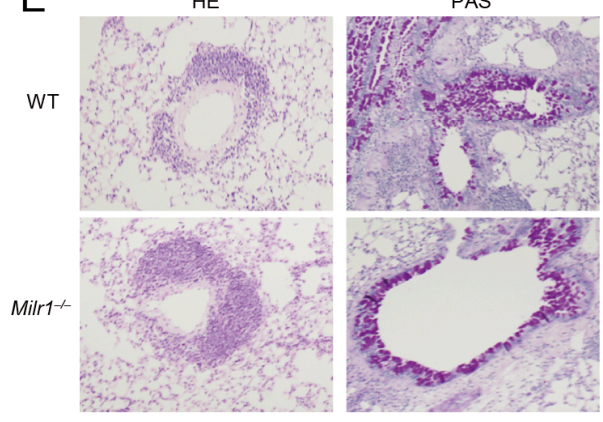

D $\quad F$
$\mathrm{F}$

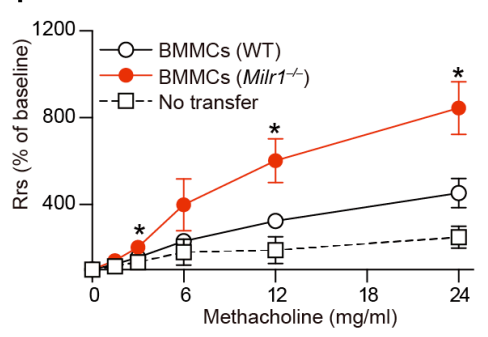

Figure 2. Allergin-1 on MCs suppresses AHR. (A) Flow cytometric analysis of MCs $\left(\mathrm{CD} 45.2^{+} \mathrm{Lin}^{-}\right.$Siglec-F $\left.{ }^{-} \mathrm{c}-\mathrm{Kit}^{+} \mathrm{Fc} \mathrm{RI}^{+}\right)$and eosinophils $\left(\mathrm{CD} 45.2^{+} \mathrm{Lin}^{-}\right.$Siglec-F $\left.\mathrm{FD}^{+} 1 \mathrm{~b}^{\text {high }}\right)$ in the lungs of WT and Milrl ${ }^{-/-}$mice after intranasal injection of HDM extract (100 $\mu \mathrm{g} / \mathrm{mouse}$ ). Blue line, WT; red line, Milr1 ${ }^{-/-}$; (right) Quantification of numbers of MCs. (B-F) DT-treated Mas-TRECK mice were injected with BMMCs derived from WT or Milr $1^{-/-}$mice. (B) Three months after the BMMCs injection, the reconstitution capacity of the injected BMMCs was assessed in the peritoneal exudate cells by flow cytometry. (C-F) DT-treated MC-reconstituted Mas-TRECK mice were immunized with HDM extract as shown in Fig. 1C. (C) BALF and (D) blood samples collected $48 \mathrm{~h}$ after the last HDM challenge were analyzed for the numbers of total cells $\left(\mathrm{CD} 45^{+}\right)$and eosinophils (CD45.2 ${ }^{+} \mathrm{Gr}-1^{\text {low }} \mathrm{CD} 11 \mathrm{c}^{-}$Siglec-F $\left.\mathrm{F}^{+}\right)$and $\mathrm{IgE}$ concentration, respectively. (E) 
Lungs were harvested $48 \mathrm{~h}$ after the last HDM challenge and analyzed for histology after treatment with hematoxylin-eosin (HE) and periodic acid-Schiff (PAS) staining. Original magnification: $\times 200$. Right, quantification of PAS-positive goblet cells. $($ F) AHR to methacholine. DT-treated Mas-TRECK mice given no BMMCs $(n=3)$ or reconstituted with BMMCs from WT $(n=4)$ or Milr1 ${ }^{-/}(n=5)$ were immunized with HDM extract. Forty-eight hours after the last HDM challenge, AHR to methacholine was analyzed. Data are representative of $3(\mathbf{C}-\mathbf{E})$ or $2(\mathbf{A}, \mathbf{B}$ and $\mathbf{F})$ independent experiments (mean \pm s.e.m.). Each point represents a single mouse. N.S., not significant. *, $P<0.05$ (WT vs Milr1 $1^{-/-}$, unpaired $t$-test). 


\section{Figure 3}
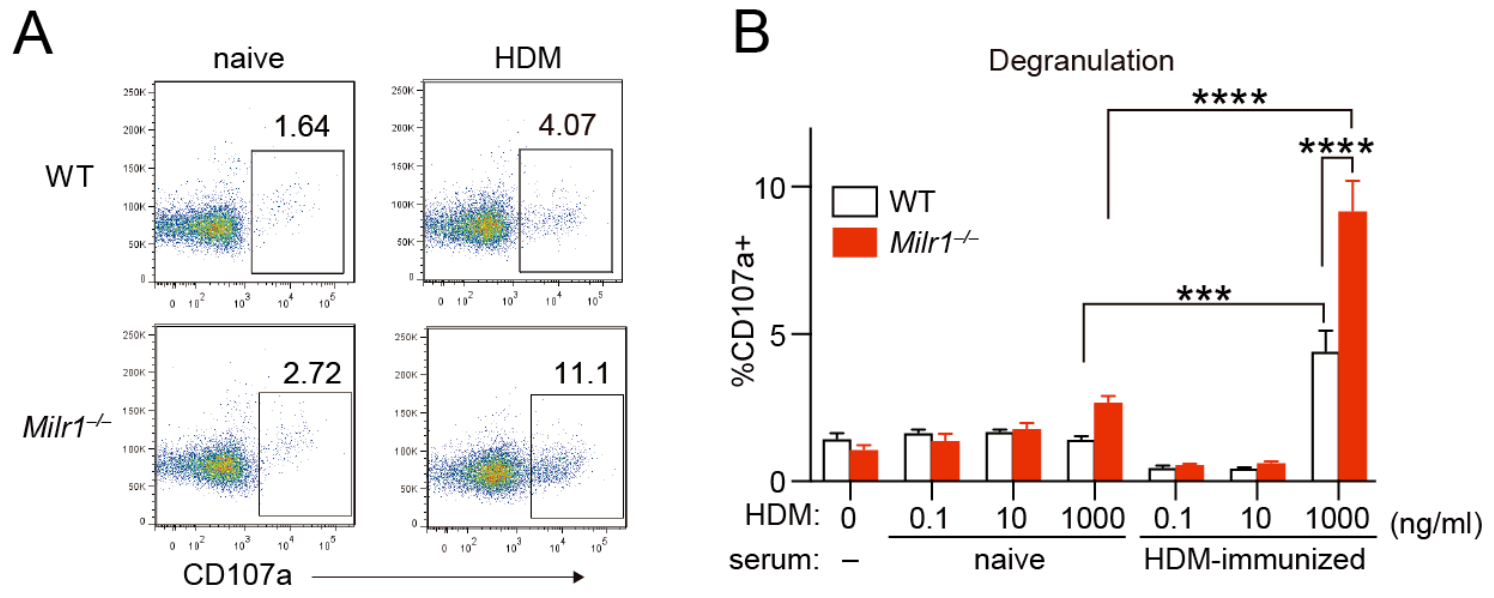

Figure 3. Allergin-1 on MCs inhibits anti-HDM antibody-dependent degranulation.

WT or Milr1 ${ }^{-/-}$BMMCs were treated for $16 \mathrm{~h}$ with serum from naïve or HDM-treated mice, followed by HDM extract stimulation for $30 \mathrm{~min}$, and analyzed for degranulation by CD107a expression. (A) representative results by flow cytometric analysis. (B) Statistical analysis. ${ }^{* * *}, P<0.005 ; * * * *, P<0.001$ (WT vs Milr $1^{-/-}$, unpaired $t$-test). Data are representative of 3 independent experiments (mean \pm s.e.m.). 